\title{
18 THE PRODNET DEMONSTRATOR
}

L.M. Camarinha-Matos ${ }^{\star}$, H. Afsarmanesh, C. Antunes, J. F. Clavier, C. Garita, P. Gibon, A. Klen, H. Lenz, C. Lima, J. Mota, A. L. Osório, R. Rabelo, H. Ribeiro, A. Schreiber, L. M. Spinosa, Y. Ugur PRODNET II Consortium

A complex virtual enterprise scenario involving European and Brazilian enterprises was designed to demonstrate and validate the approach and functionalities of the PRODNET II infrastructure. The processes involved in this scenario are summarized and selected examples illustrating the complex interactions among PRODNET modules are discussed.

\section{INTRODUCTION}

The current competitive environment asks for the enterprises to support new business and management practices. Some examples of these requirements are listed below:

- Provide faster reactions to market opportunities.

- Automatic maintenance of customer stock level through on-line monitoring.

- Optimized management of projects involving several inter-dependent suppliers.

- Provide alternatives to access, visualize and read product development specifications, without the need of specialized tools (i.e. CAD Systems).

- Management of mixed model manufacturing: Make To Stock, Assemble To Order, Make To Order, Engineer To Order, Flow Manufacturing.

\footnotetext{
- Corresponding author address: Universidade Nova de Lisboa, Quinta da Torre, 2825 Monte Caparica, Portugal, tel.: +351-1-2948517, fax: +351-1-2941253, e-mail: cam@uninova.pt.
} 
These new business practices came up in order to accomplish the recent tendencies that are guiding the SME's in the last years, such as globalization, customization and the awareness of / compliance with environmental issues.

One of the difficulties the enterprises are facing to implement these new business practices is concerned with the increasing need for faster product development processes and the use of collaborative engineering technologies. For instance: in the beginning of a product life cycle, when an enterprise receives a request to develop a new product, usually this request comes with a design specification. Sometimes the enterprise needs to change the received specification in order to adapt it to its production capabilities, and send it back again to the client. After that, the client can check and make his own changes (if needed). This interaction continues until the enterprises agree with a final and common specification. Considering this scenario, some of the current problems include the lack of the design revision control and the use of slow and not secure data communication ways (i.e. fax, e-mail).

Another difficulty is to control the relationships between two enterprises that use the Just In Time philosophy, or even deal with programmed orders. Considering the synchronization required on this business environment, any supply failure might hardly impact the production schedule. Hence the enterprises must have accurate and in-time information about all events (even the non-expected ones) to keep the schedule optimized and updated. In this case, the problems are that the current available means (i.e. fax, telephone) to support these information flows are slow, unreliable, not integrated with the legacy systems (such as the PPC) and do not allow the complete visualization and monitoring of the whole supply chain.

\section{THE PRODNET DEMONSTRATION SCENARIO}

Regarding the general enterprise requirements enumerated in the previous chapter as well as the way the PRODNET platform can tackle them, this chapter aims at presenting the general PRODNET strategy to demonstrate and validate its results. This planned demonstration focuses only on the creation and operation phase of the VE paradigm, thus the VE dissolution phase is not addressed.

For this purpose, a demonstration scenario involving 4 enterprises, 2 in Europe and 2 in Brazil was chosen. The application area is the design and manufacture of bicycles.

Demonstrating a scenario like the one envisaged by the project is actually a tough task. This arduousness is strongly related to the complexity the integration of the PRODNET platform represents in the required levels. Three basic integration levels / aspects should be considered:

i) The first one is more related to the platform's services themselves, whose execution is very complex to be managed internally. There are plenty of and different nature of actions that shall be executed in / managed by the platform, some synchronous some asynchronous, such as: the exchange of information between the platform's modules, usually in real time and cyclically; thousands of queries to a distributed / federated database; the creation of new instances of virtual enterprises by means of manual configuration of the platform; the 
interactive monitoring of the supply-chain; and the intensive use of the network in order to communicate with the other enterprises / countries.

ii) The second aspect refers to the fact that the integration obstacle is increased by the need to accommodate distributed and heterogeneous applications into a common environment and low cost hardware (PC). It comprises the integration of the platform with some legacy systems, i.e., a PPC and possibly some PDM tools in the PRODNET scenario. This means to face the hard issue of interoperation, when all the modules have to communicate with each other by means of potentially and usually different protocols and APIs.

iii) The third and final aspect refers to the intrinsic amplification of the integration and testing problems due to the inter-enterprise / intercontinental communications (through the Internet) with "almost real" transactions that shall be tackled.

Considering those three aspects, it was concluded that all those levels of integration are overwhelmingly complicated to be carried out in just one step. In the same way, the defined demonstration scenario - and the respective services involved in - is too wide to be contemplated in just one time. Thus, a more "balanced" and step-by-step strategy was adopted. The demonstrator was divided into two cases, i.e., two VEs (figure 1). The first one involving just two of the four actors involved (Órbita and Miralago), making use of only some PRODNET's modules and testing some of their services. The second one involving all the four actors (Órbita, Miralago, Akros and Herten) and using the complete set of services from all PRODNET's modules.

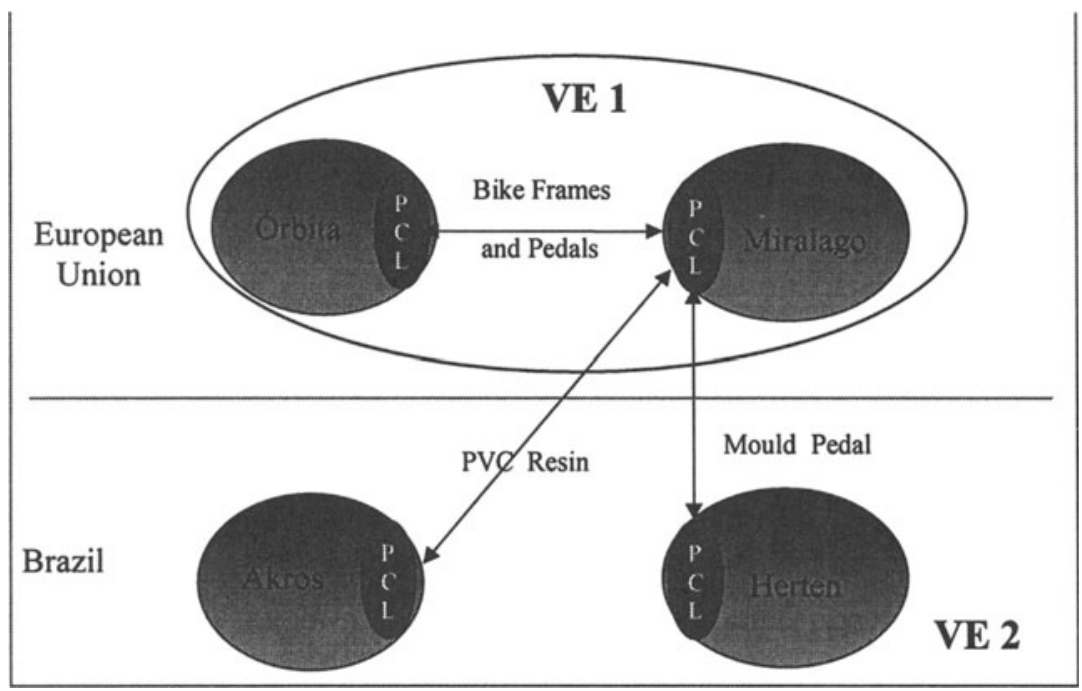

Figure 1 - PRODNET Demonstration scenario 


\section{Demonstration case 1}

This first case intends to demonstrate the operation of the basic integration mechanisms between some of the PRODNET modules.

The core strategy is to provide the consortium partners with a small but representative / real scenario, as already mentioned in this section. It represents the situation when the Órbita Company (the VE coordinator) plans to make business with the Miralago Company as a bike frame supplier. Roles of companies:

- Órbita assembles bicycles and needs to buy pieces (in this case, bike frames) in the market;

- Miralago produces and supplies bike frames.

The overall implantation methodology for this scenario comprises the gradual introduction and testing of every involved functionality. However, even for a very specific business, there are in fact several operations an enterprise perform to carry it out, according to its internal operational procedures. Even considering that they are made sometimes almost "implicitly", the PRODNET platform / services should "emulate" them.

Regarding the modules involved in this scenario and seeking an "interesting" situation within it, the "send purchase order" case was selected to be the initial testbed for the main functionalities of some modules. Five modules were involved in this first demonstration phase:

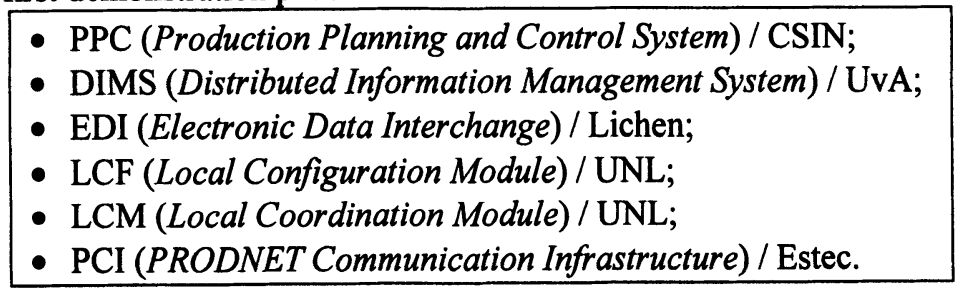

Figure 2 shows the main steps involved in this process that is supported by the infrastructures installed in each company. The various modules involved in the implementation of the various steps are also represented on the right hand side of the box representing an activity.
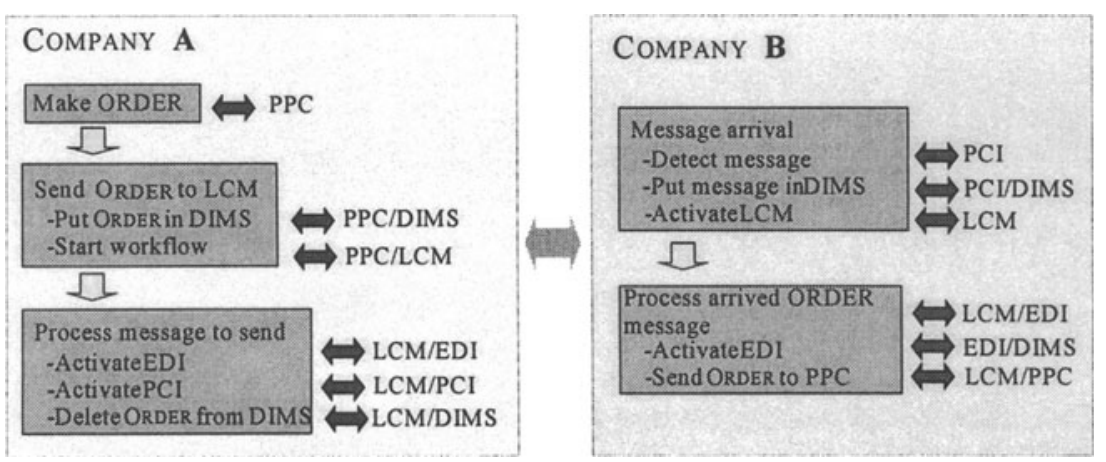

Figure 2 - Sending and receiving an order 
The workflow plans implicit in this diagram are configured via the Workflow Graphical Editor (LCF) and correspond just to one example of a possible enterprise behavior.

The implementation of this demonstration case was fundamental for testing the PRODNET modules integration approach, to test a subset of functionalities, and to validate the adequacy of the workflow-based coordination model. This simple scenario was also the basis for starting a more intense interaction with the end users towards the definition of a global business-oriented demonstration scenario.

\section{Demonstration case 2}

This case aims at demonstrating a complete business scenario, with all PRODNET modules and their functionalities being tested. Four actors are involved and the main purpose of this scenario is to produce a bike pedal. Órbita and Miralago sign a contract and start the development of a pedal, which is produced according to Órbita needs. Such pedal is produced through plastic injection and Miralago needs to find a PVC resin supplier - Akros, and a mould maker supplier - Herten, in this case. Miralago buys PVC resin from Akros. After that, Miralago and Herten exchange STEP models to produce the mould design. Finally, Miralago produces the pedal, which is approved by Órbita. The pedal production is started. Roles of companies:

- Órbita is a bike producer that needs a pedal supplier;

- Miralago is a pedal producer, that needs two suppliers: PVC resin supplier and mould maker supplier.

- Akros is a PVC resin supplier;

- Herten produces moulds.

For that purpose, the involved modules are:

- PPC (Production Planning and Control System module) / CSIN;

- DIMS (Distributed Information Management System module) / UvA;

- EDI (Electronic Data Interchange module) / Lichen;

- LCF (Local Configuration Module)/UNL;

- LCM (Local Coordination Module)/ UNL;

- PCI (Prodnet Communication Infrastructure module) / ESTEC;

- STEP (PDT module) / ProSTEP;

- DBMPS (Distributed Business Process Management System module)/ UFSC;

- EPST (Electronic Partners Search and Selection tool) / UNL.

Next sections present this scenario in detail.

\section{MAIN INTERACTIONS AMONG COMPANIES}

In order to better understand the interactions involved in this case, three groups of interoperations are involved, respectively: Órbita and Miralago, Miralago and Herten, and Miralago and Akros. 


\section{First Interoperation (between Miralago and Órbita)}

Figure 3 and the next table show the main steps involved in this interaction.

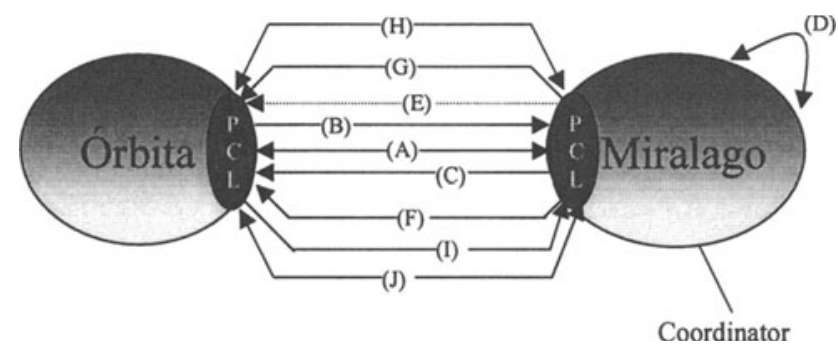

Figure 3 - Interoperation between Órbita and Miralago

\begin{tabular}{|l|l|}
\hline Steps & Modules involved \\
\hline Contract is signed; (A) & \\
\hline Orbita generates a programmed order and sends it to Miralago; (B) & $\begin{array}{l}\text { PPC, EDI, LCM, } \\
\text { PCI, DIMS }\end{array}$ \\
\hline $\begin{array}{l}\text { Order acceptance is generated by Miralago (from PPC or timed-out } \\
\text { event, based on contract rules) and sent to Órbita; }\end{array}$ & $\begin{array}{l}\text { PPC, EDI, PCI, } \\
\text { LCM, DIMS }\end{array}$ \\
\hline $\begin{array}{l}\text { Miralago's PPC generates a production order according to Órbitas's } \\
\text { order; (D) }\end{array}$ & PPC \\
\hline Miralago manages the VE orders; (J) & $\begin{array}{l}\text { DBPMS, PPC, } \\
\text { LCM, DIMS, PCI }\end{array}$ \\
\hline $\begin{array}{l}\text { Orbita can request "production follow-up" bulletins from Miralago; } \\
\text { (K) }\end{array}$ & $\begin{array}{l}\text { PPC, EDI, PCI, } \\
\text { LCM, DIMS }\end{array}$ \\
\hline Miralago delivers both pedals and delivery record to Orbita; (E, F) & $\begin{array}{l}\text { PPC, EDI, PCI, } \\
\text { LCM, DIMS }\end{array}$ \\
\hline Orbita receives and inspects the pedals; & PPC \\
\hline $\begin{array}{l}\text { Orbita sends a "reception report" to Miralago if any manufacturing } \\
\text { problem is found in the pedals; (H) }\end{array}$ & $\begin{array}{l}\text { PPC, EDI, PCI, } \\
\text { LCM, DIMS }\end{array}$ \\
\hline Miralago (PPC) generates an invoice and sends it to Orbita; (G) & PPC \\
\hline
\end{tabular}

\section{Second Interoperation (between Miralago and Akros)}

Figure 4 and the next table show the main steps involved in this interaction.

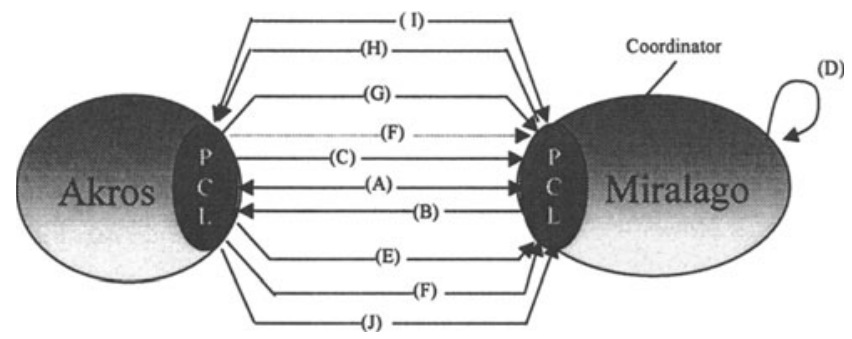

Figure 4 - Interoperation between Akros and Miralago 


\begin{tabular}{|l|l|}
\hline Steps & Modules involved \\
\hline Miralago searches a PVC resin supplier; & PST \\
\hline Miralago identifies Akros as a resin supplier; & PST \\
\hline Contract is signed and a long range supply is planned; (A) & Contract Management \\
\hline Suppliers data is configured in PPC & PPC \\
\hline Miralago generates programmed order and send it to Akros; & $\begin{array}{l}\text { PPC, EDI, PCI, LCM, } \\
\text { DIMS }\end{array}$ \\
\hline $\begin{array}{l}\text { Akros sends notification of order acceptance to Miralago (according } \\
\text { to the contract rules); (C) }\end{array}$ & $\begin{array}{l}\text { PPC, EDI, PCI, LCM, } \\
\text { DIMS }\end{array}$ \\
\hline Miralago manages the VE orders (J); & $\begin{array}{l}\text { PPC, LCM, } \\
\text { DIMS, DBPMS, PCI }\end{array}$ \\
\hline Production is started by Akros; & PPC \\
\hline Miralago can request "production follow-up" bulletins; (E) & $\begin{array}{l}\text { PPC, EDI, PCI, LCM, } \\
\text { DIMS }\end{array}$ \\
\hline Akros delivers both resin and delivery record to Miralago; (F, G) & $\begin{array}{l}\text { PPC, EDI, PCI, LCM, } \\
\text { DIMS }\end{array}$ \\
\hline Akros (PPC system) generates an invoice and sends it to Miralago; & $\begin{array}{l}\text { PPC, EDI, PCI, LCM, } \\
\text { DIMS }\end{array}$ \\
\hline Miralago receives and inspects the product; & PPC \\
\hline $\begin{array}{l}\text { Miralago sends a "reception report" to Akros if any manufacturing } \\
\text { problem is found in the product; (H) }\end{array}$ & $\begin{array}{l}\text { PPC, EDI, PCI, LCM, } \\
\text { DIMS }\end{array}$ \\
\hline
\end{tabular}

\section{Third Interoperation (between Miralago and Herten)}

Figure 5 and the next table show the main steps involved in this interaction.

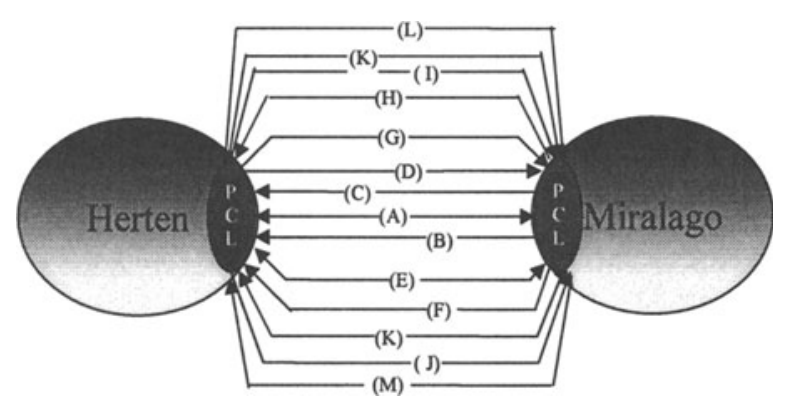

Figure 5 - Interoperation between Herten and Miralago

\begin{tabular}{|l|l|}
\hline Steps & Modules involved \\
\hline Miralago searches a mould maker supplier; & PST \\
\hline Miralago identifies Herten as a supplier; & PST \\
\hline $\begin{array}{l}\text { An industrial "confidential" contract is signed, and the VE is } \\
\text { configured; }\end{array}$ \\
\hline Miralago sends a pedal design to Herten; (C) & $\begin{array}{l}\text { STEP,LCM,EDI, } \\
\text { PCI,DIMS }\end{array}$ \\
\hline $\begin{array}{l}\text { Herten and Miralago analyze interactively the project, exchanging } \\
\text { STEP models; (E) }\end{array}$ & $\begin{array}{l}\text { STEP,LCM,EDI, } \\
\text { PCI,DIMS }\end{array}$ \\
\hline
\end{tabular}




\begin{tabular}{|l|l|}
\hline $\begin{array}{l}\text { Miralago agrees with the design and sends a "design acceptance" to } \\
\text { Herten; (F) }\end{array}$ & $\begin{array}{l}\text { STEP,LCM,EDI, } \\
\text { PCI,DIMS }\end{array}$ \\
\hline Herten sends proposal for the mould production; (G) & \\
\hline $\begin{array}{l}\text { Miralago evaluates the proposal and sends a "confirmation order" to } \\
\text { Herten; (H) }\end{array}$ & \\
\hline Contract is signed and sporadic supply is planned; (A) & $\begin{array}{l}\text { Contract } \\
\text { Management }\end{array}$ \\
\hline Suppliers and product data are configured in PPC; & PPC \\
\hline Miralago generates and order entry and sends it to Herten; (B) & $\begin{array}{l}\text { PPC, EDI, PCI, } \\
\text { LCM, DIMS }\end{array}$ \\
\hline Herten sends an order acceptance and the project confirmation; (D) & $\begin{array}{l}\text { PPC, EDI, PCI, } \\
\text { LCM, DIMS }\end{array}$ \\
\hline Production is started by Herten; & PPC \\
\hline Miralago manages the VE orders; (N) & $\begin{array}{l}\text { PPC, LCM, DIMS, } \\
\text { DBPMS, PCI }\end{array}$ \\
\hline Miralago can request "production follow up bulletins" from Herten; (I) & $\begin{array}{l}\text { PPC, EDI, PCI, } \\
\text { LCM, DIMS, ACF }\end{array}$ \\
\hline Herten delivers pedal mould and delivery record to Miralago; (J,K) & $\begin{array}{l}\text { PPC, EDI, PCI, } \\
\text { LCM, DIMS }\end{array}$ \\
\hline Miralago receives and inspects the product; & PPC \\
\hline Miralago sends a "reception report" to Herten; (L) & $\begin{array}{l}\text { PPC, EDI, PCI, } \\
\text { LCM, DIMS }\end{array}$ \\
\hline
\end{tabular}

\section{A TIGHT INTEGRATION PHILOSOPHY}

The PRODNET infrastructure, although based on several independent modules, presents a very tight integration of its key components. This integration will be illustrated using two examples. The first one is the interaction " $\mathrm{N}$ " from the previous scenario, which involves "Miralago manages VE orders". This single interaction actually involves the execution of several PCL core services that request auxiliary services from a number of PCL modules and enterprise applications, namely the DBPMS, DIMS, LCM, PCI, and PPC.

The process starts at Miralago, the VE coordinator, when its DBPMS module wants to monitor/manage the execution of orders. According to its needs, DBPMS activates the execution of one of the following core services: PCL_GetRequestedOrder, PCL_GetReqOrdTree, PCL_GetRequestedItem or PCL_GetProductionOrder (Figure 6, action 1). LCM starts executing the workflow plan defined for the requested core service, i.e. the LCM calls the auxiliary services involved in the plan (action 2). In this example, DIMS is the provider of the auxiliary services. DIMS on its turn, must perform several actions in order to get the information requested by LCM. These actions indeed represent the federated query process (FQP) that may involve the local PPC and/or the PPC of remote VE partners (action 3). When DIMS receives the requested information, it sends back to LCM the results related to the previous request (action 4). Finally, LCM is able to send back to DBPMS, the results of the requested core service (action 5). 


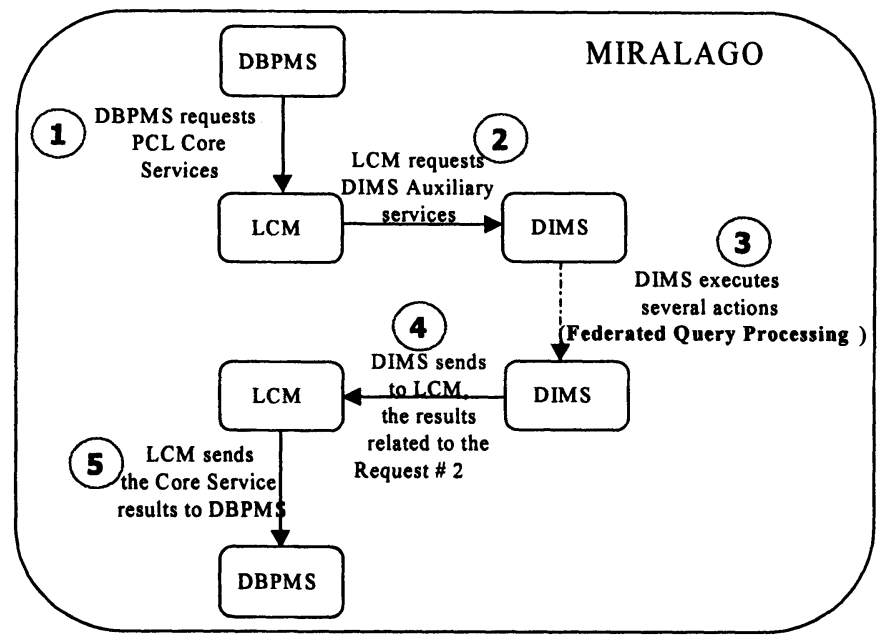

Figure 6 - Monitoring an order

In general the FQP, managed by DIMS, can involve the execution of another group of PCL core services. In order to answer the LCM request (action 2), DIMS may need to collect information from the PPCs running in other VE members (Akros, Herten and Orbita). This also involves the communication processes provided by PCI and the execution of some core services, supported by LCM.

For this example, the FQP is composed of the following steps: DIMS requests from LCM the execution of the following core services: PCL_SendInternalDimsQuery (case i.), PCL_SendExternalDimsQuery (case ii.). In the case i, (Figure 7A), LCM calls the PPC_PutData auxiliary service from the PPC module. The PPC performs some actions and sends back an answer to LCM, which is able to send back the core service results to DIMS. In the case ii, LCM calls the PCI_DeliverMessage auxiliary service from PCI, which sends a message out to a certain partner. After receiving the results of this communication process, PCI sends back an answer to LCM, and LCM sends the core service results to DIMS.

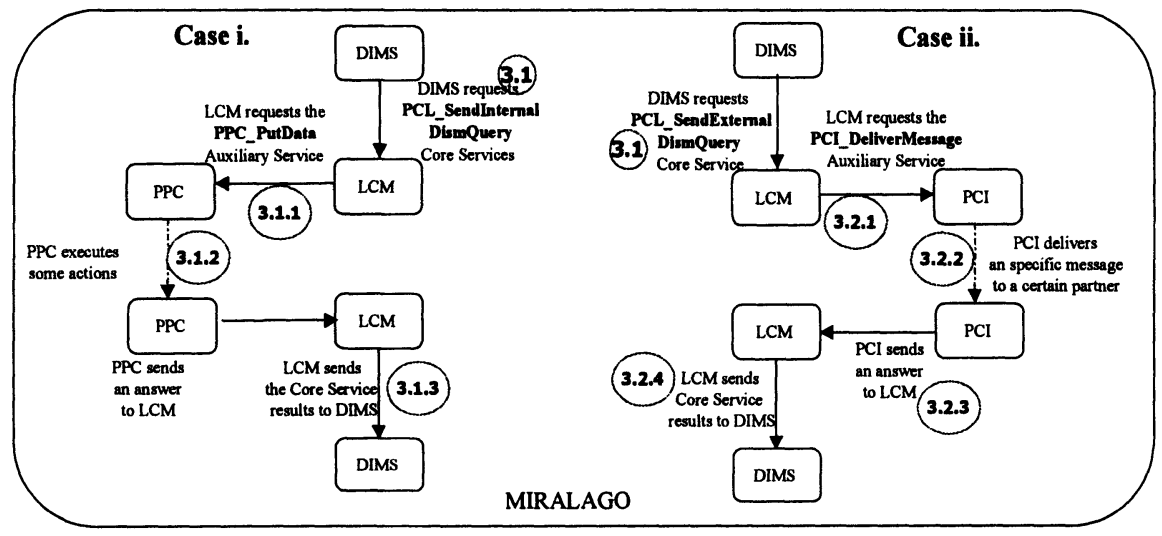

Figure 7A - PCL_SendInternal DimsQuery

Figure 7B - PCL_SendExternal DimsQuery 


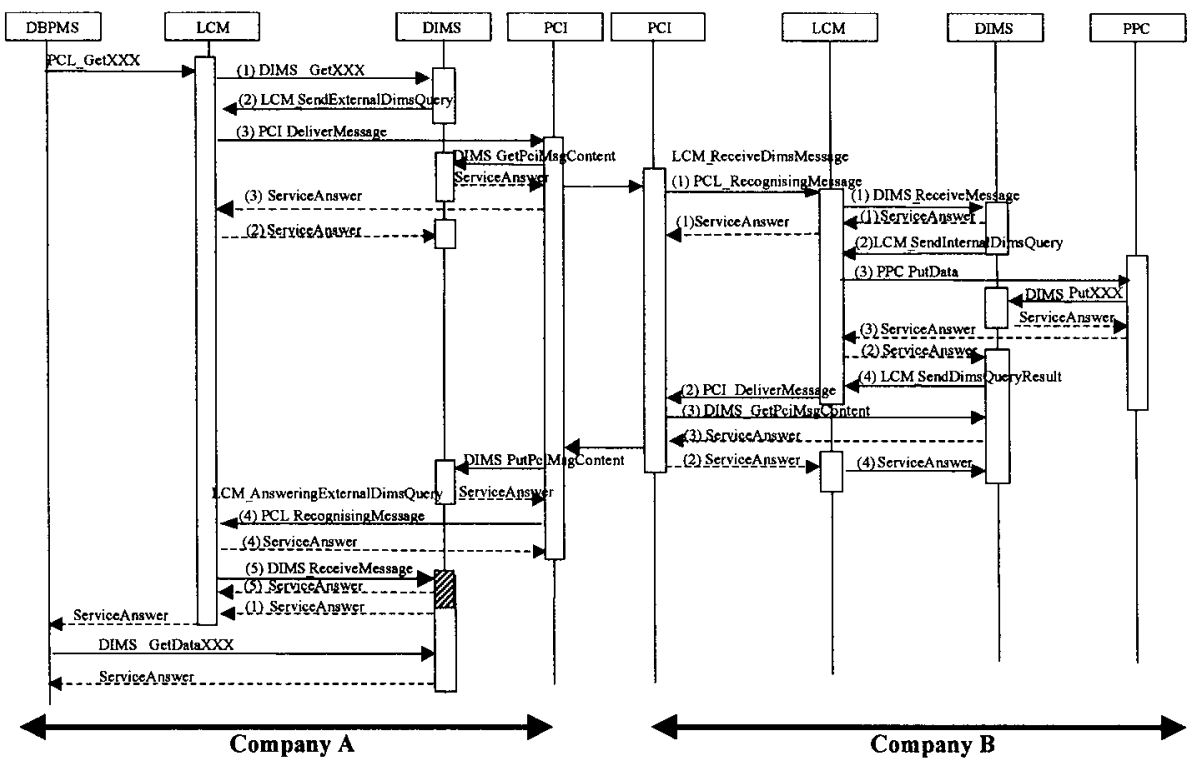

Figure 8 - Example of the steps involved in a federated query process

Figure 8 presents the complete view of all services (core and auxiliary) involved in this FQP. In order to support the coordination of all these steps, several workflow plans are defined in each enterprise. Figure 9 shows just a part of the workflow model built for supporting MIRALAGO operation, in the demonstration scenario.

The second example (Figures 10 and 11), also extracted from the third interoperation, is based on the interactions $\mathrm{C}$ and $\mathrm{E}$ defined earlier that are "Miralago sends a pedal design to Herten" and "Herten and Miralago analyze interactively the project, exchanging STEP models".

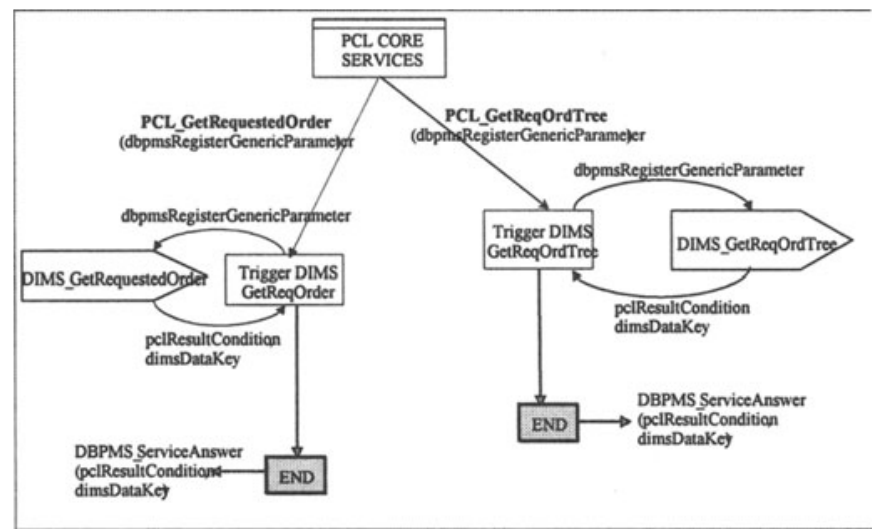

Figure 9 - Partial example of workflow model 


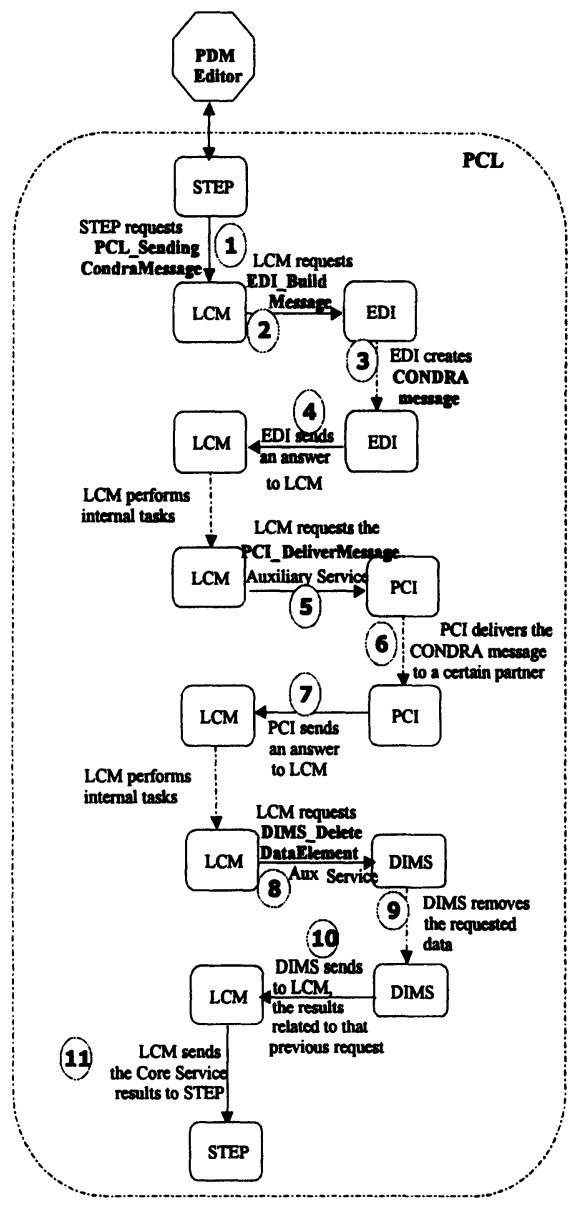

Figure 10 - Sending a CONDRA message

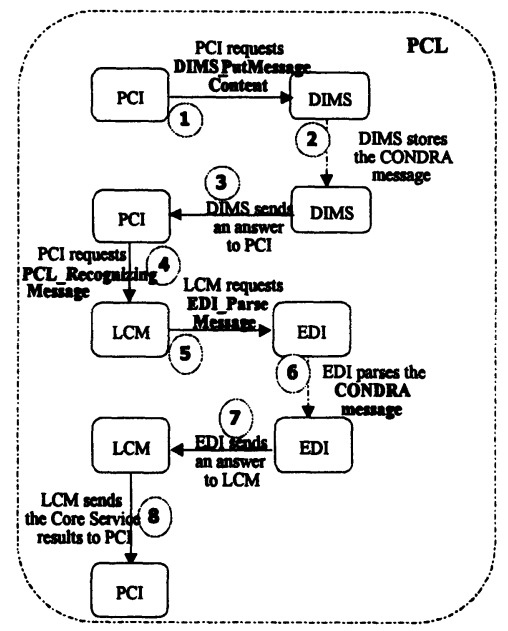

Figure 11a-Receiving and parsing a CONDRA message

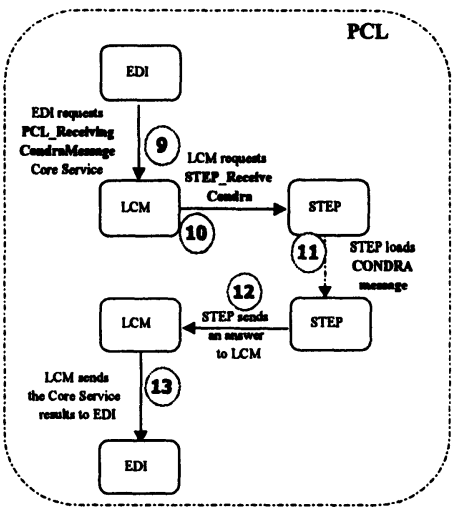

Figure $11 \mathrm{~b}$ - Sending a CONDRA message to STEP

These steps are intended to support the product design negotiation process to be carried out between Miralago and Herten, which indeed is based on the exchange of product models written in STEP format. The PCL modules involved in this process are STEP, LCM, EDI, DIMS and PCI. A PDM editor is also involved in this process. The product negotiation is started at the PDM editor with the creation of the PDM data. The PDM editor calls the STEP module, which activates the execution of the PCL_SendingCondraMessage.

These examples illustrate the complexity of the interactions among the various modules of the PRODNET platform. All critical coordination aspects are handled through the workflow plans that are easily designed via the graphical workflow editor. 


\section{CONCLUSIONS}

A complex demonstration scenario was developed to demonstrate and assess the infrastructure proposed by PRODNET II. This scenario considers a virtual enterprise composed of European and Brazilian enterprises and is based on real business needs as formulated by end user companies.

The developed infrastructure proved to be flexible enough to support such scenario. This scenario fulfills the main goal of PRODNET II, that was the design and implementation of an open and flexible infrastructure for virtual enterprises, specially focused on the needs of small and medium size enterprises.

Among the major features demonstrated, the following can be emphasized:

- Support for information exchange based on standards such as EDIFACT and STEP.

- Safe communications, guaranteeing privacy and authentication ver a variety of protocols.

- Federated / distributed information management coping with the privacy and information visibility needs of the VE and each of its individual members.

- Support for flexible and easily configurable coordination mechanisms based on advanced hierarchical workflow mechanisms.

- Distributed business processes management and monitoring.

- Support for the VE creation and reconfiguration through partners' search and configuration tools.

- Extensions to legacy PPC system supporting reactive behavior in the VE environment and offering quality and imprecise and incomplete orders management along the supply chain.

Although composed by a large number of independent modules developed by different partners, the PRODNET infrastructure features a tight integration approach, a requirement for a truly flexible infrastructure.

\section{Acknowledgements}

This work was funded in part by the European Commission, Esprit programme, and the Brazilian research council $(\mathrm{CNPq})$. The authors also thank the valuable contributions from their partners of the PRODNET II consortium: CSIN (P), HERTEN (BR), MIRALAGO (P), University of Amsterdam (NL), Universidade Federal de Santa Catarina (BR), Universidade Nova de Lisboa (P), Lichen Informatique (F), ProSTEP (Germany), Uninova (P), ESTEC (P).

\section{REFERENCES}

1. Camarinha-Matos, L.M.; Afsarmanesh, H.; Lima, C. - Hirerachical coordination in virtual enterprise infrastructures, to appear in Journal of Intelligent and Robotic Systems, 1999.

2. Camarinha-Matos, L.M.; Afsarmanesh, H.; Garita, C.; Lima, C. - Towards an architecture for virtual enterprisesJournal of Intelligent Manufacturing, Vol. 9, Issue 2, Abr 1998, pp 189-199. 